\title{
Asymptotic Farkas lemmas for convex
}

\section{systems}

\section{- Nguyen Dinh}

University International, VNU - HCM

\section{- Tran Hong Mo}

Tien Giang University, Tien Giang

(Received on June $5^{\text {th }} 2015$, accepted on November $21^{\text {th }} 2016$ )

\section{ASTRACT}

In this paper we establish characterizations of the containment of the set $\{x \in X: x \in C, g(x) \in-K\} \subset\{x \in X: f(x) \geq 0\}$, where $C$ is a closed convex subset of a locally convex Hausdorff topological vector space, $X, K$ is a closed convex cone in another locally convex Hausdorff topological vector space and $g: X \rightarrow Y$ is a $K$ - convex mapping, in a reverse convex set, define by the proper, lower semicontinuous, convex function. Here, no constraint qualification condition or qualification condition are assumed. The characterizations are often called asymptotic Farkas-type results. The second part of the paper was devoted to variant Asymptotic Farkas-type results where the mapping is a convex mapping with respect to an extended sublinear function. It is also shown that under some closedness conditions, these asymptotic Farkas lemmas go back to non-asymptotic Farkas lemmas or stable Farkas lemmas established recently in the literature. The results can be used to study the optimization

Keywords: Farkas lemma, sequential Farkas lemma, limit inferior, limit superior

\section{INTRODUCTION AND PRELIMINARIES}

Farkas-type results have been used as one of the main tools in the theory of optimization [8]. Typical Farkas lemma for cone-convex systems characterizes the containment of the set where is a closed convex subset of a locally convex Hausdorff topological vector space (brieftly, lcHtvs), is a closed convex cone in another lcHtvs and is a convex mapping, in a reverse convex set, define by the proper, lower semi-continuous, convex function. If the characterization holds under some constraint qualification condition or qualification condition then it is called non-asymptotic Farkas-type result (see [6], [10-12]). Otherwise (i.e., without any qualification condition), such characterizations often hold in the limit forms and they are called asymptotic Farkas-type results (see [7, 5, 9, 13] and references therein). In this paper, we mainly established several forms of asymptotic Farkas-type results for convex systems in the two means: systems convex with respect to a convex cone (called -convex systems) and systems convex w.r.t. an extended sublinear function $S$ (called $S$-convex systems). The results can be used to establish the optimality conditions and dulaity for optimization problems where constraint qualification conditions failed, such as classes of semidefinite programs, or scalarized multi-objective programs, scalarized vector optimization problems. We shoned also that under some closedness conditions, these asymptotic Farkas lemmas came back to non-asymptotic Farkas lemmas or stable Farkas lemmas established recently in the literature.

Let $X$ and $Y$ be lcHtvs, with their topological dual spaces $X^{*}$ and $Y^{*}$, respectively. The only topology we consider on $X^{*}, Y^{*}$ is the weak* topology. For a set $A \subset X^{*}$, the closure of $A$ w.r.t. 
the weak $^{*}$-topology is denoted by $\operatorname{cl} A$. The indicator function of $A$ is denoted by $i_{A}$, i.e., $i_{A}(x)=0$ if $x \in A, i_{A}(x)=+\infty$ if $\quad x \in X \backslash A$. Let $f: X \rightarrow \overline{\mathbb{R}} \cup\{ \pm \infty\}$. The effective domain of $f$ is the set $\operatorname{dom} f:=\{x \in X: f(x)<+\infty\}$. The function $f$ is proper if $\operatorname{dom} f \neq \varnothing$. The set of all proper, lower semi-continuous (lsc in short) and convex functions on $X$ will be denoted by $\Gamma(X)$. The epigraph of $f$ is

$$
\text { epi } f:=\{(x, \alpha) \in X \times \overline{\mathbb{R}}: f(x) \leq \alpha\}
$$

The Legendre-Fenchel conjugate of $f$ is the function $f^{*}: X^{*} \rightarrow \overline{\mathbb{R}}:=\overline{\mathbb{R}} \cup\{ \pm \infty\}$ defined by

$$
f^{*}\left(x^{*}\right)=\sup _{x \in X}\left\{\left\langle x^{*}, x\right\rangle-f(x)\right\}, \forall x^{*} \in X^{*} .
$$

It is clear that for any $x^{*} \in X^{*}$ and $\bar{x} \in X$, the Young-Fenchel inequality always holds:

$$
f^{*}\left(x^{*}\right) \geq\left\langle x^{*}, \bar{x}\right\rangle-f(\bar{x}) \text {. }
$$

Moreover, for any $\beta \in \mathbb{R}$ one has $(f-\beta)^{*}\left(x^{*}\right)=f^{*}\left(x^{*}\right)+\beta$ for all $x^{*} \in X^{*}$.

Now let $K$ be a closed convex cone in $Y$ and let $\leq_{K}$ be the partial order on $Y$ generated by $K$, i.e., $y_{1} \leq_{K} y_{2}$ if and only if $y_{2}-y_{1} \in K$.

We add to $Y$ a greatest element with respect to $\leq_{K}$, denoted by $\infty_{K}$, which does not belong to $Y$, and let $Y^{\bullet}=Y \cup\left\{\infty_{K}\right\}$.

Then one has $y \leq_{K} \infty_{K}$ forevery $y \in Y^{*}$. We assume by convention: $y+\infty_{K}=\infty_{K}+y=\infty_{K}$, for all $y \in Y^{*}$, and $\alpha \infty_{K}=\infty_{K}$ if $\alpha \geq 0$. The dual cone of $K$, denoted by $K^{+}$, is defined by $K^{+}:=\left\{y^{*} \in Y^{*}:\left\langle y^{*}, y\right\rangle \geq 0, \forall y \in K\right\}$. A mapping $h: X \rightarrow Y^{\bullet}$ is called $K$-convex if

$x_{1}, x_{2} \in X, \mu_{1}, \mu_{2}>0, \mu_{1}+\mu_{2}=1$

$\Rightarrow h\left(\mu_{1} x_{1}+\mu_{2} x_{2}\right) \leq_{K} \mu_{1} h\left(x_{1}\right)+\mu_{2} h\left(x_{2}\right)$,

where $" \leq_{K}$ " is the binary relation (generated by $K$ ) extended to $Y^{\bullet}$ by setting $y \leq_{K} \infty_{K}$ forall $y \in Y^{\bullet}$. The domain of $h$, denoted by $\operatorname{dom} h$, is defined to be the set $\operatorname{dom} h:=\{x \in X: h(x) \in Y\}$.

The $K$-epigraph of $h$ is the set epi $_{K} h:=\{(x, y) \in X \times Y: y \in h(x)+K\}$. space. Then, $h^{-1}(-K)$ is closed (see [6]). It is worth observing that if $h$ is $K$-convex, then $h^{-1}(-K)$ is convex. Moreover, for any $y^{*} \in Y^{*}$ and $g: X \rightarrow Y^{\bullet}$, we define the composite function $y^{*} o g: X \rightarrow \mathbb{R} \cup\{+\infty\}$ as follows

$$
\left(y^{*} \circ g\right)(x)= \begin{cases}\left\langle y^{*}, g(x)\right\rangle, & \text { if } x \in \operatorname{dom} g, \\ +\infty, & \text { else. }\end{cases}
$$

The function $S: Y \rightarrow \mathbb{R} \cup\{+\infty\}$ is called (extended) sublinear if

$$
\begin{aligned}
& S\left(y+y^{\prime}\right) \leq S(y)+S\left(y^{\prime}\right), \\
& \text { and } S(\lambda y)=\lambda S(y), \forall y, y^{\prime} \in Y, \forall \lambda>0
\end{aligned}
$$

By convention, we set $S\left(0_{Y}\right)=0$ (this convention is appropriate to the assumption that $S$ is lsc). Such a function $S$ can be extended to $Y^{\bullet}$ by setting $S\left(\infty_{K}\right)=+\infty$. An extended sublinear function $S: Y \rightarrow \mathbb{R} \cup\{+\infty\}$ allows us to introduce in $Y^{\bullet}$ a binary relation which is reflexive and transitive:

$$
\begin{aligned}
& y_{1} \leq_{S} y_{2} \text { if } y_{1} \leq_{K} y_{2}, \\
& \text { where } K=\{y \in Y: S(-y) \leq 0\}
\end{aligned}
$$

and $y \leq_{S} \infty_{K}$ for all $y \in Y^{\bullet}$. We consider also the extension of $\mathrm{S}$ as $S: Y^{\bullet} \rightarrow \mathbb{R} \cup\{+\infty\}$ By setting $S\left(\infty_{K}\right)=+\infty$.

Given an extended sublinear function $S: Y \rightarrow$ $\mathbb{R} \cup\{+\infty\}$, we adapt the notion $S$-convex

( i.e., convex with respect to a sublinear function) in [6] which generalized the one in [16].

It is clear that $h$ is $K$-convex if and only if epi $_{K} h$ is convex. In addition, $h: X \rightarrow Y^{\bullet}$ is said to be $K$-epi closed if epi $_{K} h$ is a closed set in the product A mapping $h: X \rightarrow Y^{\bullet}$ is said to be $S$-convex if for all $x_{1}, x_{2} \in X, \mu_{1}, \mu_{2}>0, \mu_{1}+\mu_{2}=1$, one has

$$
h\left(\mu_{1} x_{1}+\mu_{2} x_{2}\right) \leq_{S} \mu_{1} h\left(x_{1}\right)+\mu_{2} h\left(x_{2}\right) .
$$

It is worth observing that, as mentioned in [15, Remark 1.10], " $S$ - convex means different things

under different circumstances" such as, when $Y=\mathbb{R}$, if $S(y):=|y|, \quad S(y):=y, \quad S(y):=$, or $S(y)=0$, respectively, then " $S$-convex" means 
"affine", "convex", "concave" or "arbitrary", respectively.

Moreover, the equalities hold whenever one of the nets is convergent.

It is clear that if $h$ is $S$-convex then $h$ is $K$ convex with $K:=\{y \in Y: S(-y) \leq 0\}$. Conversely, if $h$ is $K$-convex with some convex cone $K$ then $h$ is $S$-convex with $S=i_{-K}$ (see [6]).

Definition 1.1 [2, p.5] [1, p.32], [14, p.217] Let $\left(a_{i}\right)_{i \in I}$ be a net of extended real numbers defined on a directed set $(\mathrm{I}, \gg) e$ define limit inferior of the net $\left(a_{i}\right)_{i \in I}$ as follows

$$
\liminf _{i \in I} a_{i}:=\liminf _{i \in I} a_{j}=\sup _{i \in I} \inf _{j{ }_{i}} a_{j}
$$

Similarly, limit superior of the net $\left(a_{i}\right)_{i \in I}$ is defined by

$$
\underset{i \in I}{\limsup } a_{i}:=\limsup _{i \in I} a_{j}=\inf _{i \in I} \sup _{j ? i} a_{j} .
$$

We say that $\left(a_{i}\right)_{i \in I}$ converges to $a \in \mathbb{R}$ denoted by $\lim a_{i}=a$ or $a_{i} \rightarrow a$, if for any $\varepsilon>0$, there exists $i \in i_{0} \in I$ such that $\left|a_{i}-a\right|<\varepsilon$ for all $i \gg i_{0}$

The following properties were given in [2, p.9] and [14, p.221].

Lemma 1.1 Let $\left(a_{i}\right)_{i \in I}$ and $\left(b_{i}\right)_{i \in I}$ be nets of extended real numbers. Then the following statements hold:

(i)

$\underset{i \in I}{\limsup }\left(-a_{i}\right)=-\liminf _{i \in I} a_{i}$ and $\limsup _{i \in I} a_{i} \geq \liminf _{i \in I} a_{i}$.

(ii) $\lim _{i \in I} a_{i}=a \in \mathrm{i}$ if and only if

$$
\liminf _{i \in I} a_{i}=\limsup _{i \in I} a_{i}=a .
$$

(iii) If $a_{i} \leq b_{i}$ for all $i \in I$, then

$\underset{i \in I}{\liminf } a_{i} \leq \liminf _{i \in I} b_{i}$ and $\limsup _{i \in I} a_{i} \leq \limsup _{i \in I} b_{i}$.

$$
\liminf _{i \in I}\left(a_{i}+b_{i}\right) \geq \liminf _{i \in I} a_{i}+\liminf _{i \in I} b_{i},
$$

and $\limsup _{i \in I}\left(a_{i}+b_{i}\right) \leq \limsup _{i \in I} a_{i}+\limsup _{i \in I} b_{i}$,

provide that the right side of the inequalities are defined.
Approximate Farkas lemma for cone-convex systems

In this section we will establish one of the main result of this paper: the asymptotic version of Farkas lemma for convex systems, which holds without any qualification condition.

Let $X, Y$ lcHtvs, $K$ be a closed convex cone in $Y, C$ be a nonempty closed convex subset of $X$ and $f: X \rightarrow \mathbb{R} \cup\{+\infty\}$ be a proper lsc and convex function. Let further $g: X \rightarrow Y^{\bullet}$ be a $K$-convex and $K$-epi closed mapping. Let $A:=C \cap g^{-1}(-K) \quad$ and assume that $(\operatorname{dom} f) \cap A \neq \varnothing$.

Theorem 2.1 [Asymptotic Farkas lemma 1] The following statements are equivalent: (i) $x \in C, g(x) \in-K \Rightarrow f(x) \geq 0$,

(ii) there exist nets $\left(y_{i}^{*}\right)_{i \in I} \subset K^{+}$and $\left(x_{l i}^{*}, x_{2 i}^{*}, x_{3 i}^{*}, \varepsilon_{i}\right)_{i \in I} \subset X^{*} \times X^{*} \times X^{*} \times \mathbb{R}$ such such that

$\varepsilon_{i} \geq f^{*}\left(x_{1 i}^{*}\right)+\left(y_{i}^{*} \mathrm{og}\right)^{*}\left(x_{2 i}^{*}\right)+i_{C}^{*}\left(x_{3 i}^{*}\right), \quad \forall i \in I$

and $\left(x_{1 i}^{*}+x_{2 i}^{*}+x_{3 i}^{*}, \varepsilon_{i}\right) \rightarrow\left(0_{X^{*}}, 0\right)$,

(iii) there exist nets $\left(y_{i}^{*}\right)_{i \in I} \subset K^{+}$and

$\left(x_{i}^{*}, \varepsilon_{i,}\right)_{i \in I} \subset X^{*} \times \mathbb{R}$ such that

$\varepsilon_{i} \geq\left(f+y_{i}^{*} \mathrm{og}+i_{C}\right)^{*}\left(x_{i}^{*}\right), \quad \forall i \in I$

and

$$
\left(x_{i}^{*}, \varepsilon_{i}\right) \rightarrow\left(0_{X^{*}}, 0\right),
$$

(iv) there exists a net $\left(y_{i}^{*}\right)_{i \in I} \subset K^{+}$such that $f(x)+\liminf _{i \in I}\left(y_{i}^{*} \circ g\right)(x) \geq 0, \forall x \in C$.

Proof. [(i) $\Leftrightarrow$ (ii)] Assume that (i) holds. Observe firstly that $A$ is closed and convex. Secondly, (i) is equivalent to

$$
0 \geq\left(f+i_{A}\right)^{*}\left(0_{X^{*}}\right),
$$

or equivalently, $\left(0_{x^{*}}, 0\right) \in \operatorname{epi}\left(f+i_{A}\right)^{*}$.

Since we also have $[4$, p. 328]

$\operatorname{epi}\left(f+i_{A}\right)^{*}=\operatorname{cl}\left(\right.$ epi $\left.f^{*}+\underset{\lambda \in K^{+}}{\bigcup} \operatorname{epi}(\lambda g)^{*}+\operatorname{epi} i_{C}^{*}\right)$,

and so, (i) is equivalent to

$\left(0_{X^{*}}, 0\right) \in \mathrm{cl}\left(\operatorname{epi} f^{*}+\bigcup_{\lambda \in K^{+}} \operatorname{epi}(\lambda g)^{*}+\operatorname{epi}_{C}^{*}\right)$,

and the equivalence between (i) and (ii) follows 
[(ii) $\Rightarrow$ (iii)] Assume that (ii) holds, i.e., there exist nets $\left(y_{i}^{*}\right)_{i \in I} \subset K^{+}$and

$\left(x_{l i}^{*}, x_{2 i}^{*}, x_{3 i}^{*}, \varepsilon_{i}\right)_{i \in I} \subset X^{*} \times X^{*} \times X^{*} \times \mathbb{R}$ such that $\varepsilon_{i} \geq f^{*}\left(x_{1 i}^{*}\right)+\left(y_{i}^{*} \mathrm{o} g\right)^{*}\left(x_{2 i}^{*}\right)+i_{C}^{*}\left(x_{3 i}^{*}\right), \forall i \in I,(2.1)$ and $\left(x_{1 i}^{*}+x_{2 i}^{*}+x_{3 i}^{*}, \varepsilon_{i}\right) \rightarrow\left(0_{x^{*}}, 0\right)$.

By the definition of the conjugate function, (2.1) implies that $\varepsilon_{i} \geq\left\langle x_{1 i}^{*}+x_{2 i}^{*}+x_{3 i}^{*}, x\right\rangle-\left(f+y_{i}^{*} \mathrm{og}+i_{C}\right)(x), \forall x \in X, \forall i \in I$. Set $x_{i}^{*}:=x_{1 i}^{*}+x_{2 i}^{*}+x_{3 i}^{*}$ for all $i \in I$. Then the above inequality gives rise to

$$
\varepsilon_{i} \geq\left(f+y_{i}^{*} \mathrm{o} g+i_{C}\right)^{*}\left(x_{i}^{*}\right), \forall i \in I
$$

and (2.2) becomes $\left(x_{i}^{*}, \varepsilon_{i}\right) \rightarrow\left(0_{X^{*}}, 0\right)$.

$$
[(\mathrm{iii}) \Rightarrow \text { (iv)] Assume that (iii) holds, i.e., }
$$

there exist nets $\left(y_{i}^{*}\right)_{i \in I} \subset K^{+}$and $\left(x_{i}^{*}, \varepsilon_{i,}\right)_{i \in I} \subset$ $X^{*} \times \mathbb{R}$ such that

$$
\varepsilon_{i} \geq\left(f+y_{i}^{*} \mathrm{og}+i_{C}\right)^{*}\left(x_{i}^{*}\right), \quad \forall i \in I
$$

and

$$
\left(x_{i}^{*}, \varepsilon_{i}\right) \rightarrow\left(0_{X^{*}}, 0\right) .
$$

Again by the definition of the conjugate function, one has

$\varepsilon_{i} \geq\left\langle x_{i}^{*}, x\right\rangle-\left(f+y_{i}^{*} \mathrm{o} g+i_{C}\right)(x), \forall x \in X, \forall i \in I$,

or equivalently,

$f(x)+\left(y_{i}^{*} \circ g\right)(x) \geq\left\langle x_{i}^{*}, x\right\rangle-\varepsilon_{i}, \quad \forall x \in C, \forall i \in I$,

(which still holds even in case $x \notin \operatorname{dom} f$ and $x \notin \operatorname{dom} g$ ).

Taking liminf in both sides of the last inequality, we get (iv).

[(iv) $\Rightarrow$ (i)] Assume that (iv) holds, i.e., there exists a net $\left(y_{i}^{*}\right)_{i \in I} \subset K^{+}$such that

$f(x)+\liminf _{i \in I}\left(y_{i}^{*} \mathrm{o} g\right)(x) \geq 0, \quad \forall x \in C$.

Observe that if $x \in C$ such that $g(x) \in-K$, then $\left(y_{i}^{*} \mathrm{o} g\right)(x) \leq 0$ for all $i \in I$. Thus, for $x \in C$ such that $g(x) \in-K$, one gets

$f(x) \geq f(x)+\liminf _{i \in I}\left(y_{i}^{*} \circ g\right)(x) \geq 0$.

The proof is complete.

Remark 2.1 The equivalence $[(i) \Leftrightarrow(i v)]$ was established in [5] involved the space $Y$ (instead of $\left.Y^{\bullet}\right)$, under the assumption that $y^{*} \mathrm{o} g \in \Gamma(X)$ for all $y^{*} \in K^{+}$, which is much stronger the $S$-epi closedness of $g$ used in Theorem 2.1.

$$
\begin{aligned}
& \text { We now set } \\
& D:=\text { epi } f^{*}+\mathrm{U} \text { epi }\left(y^{*} \text { o } g\right)^{*}+\operatorname{epi} i_{C}^{*} \quad \text { and } \\
& F:=\bigcup_{y^{*} \in K^{+}} \operatorname{epi}\left(f+y^{*} \text { o } g+i_{C}\right)^{*} .
\end{aligned}
$$

From the proof of Theorem 2.1, we get

Corollary 2.1 [Farkas lemma for cone-convex systems] Consider the following conditions:

$$
\begin{gathered}
\left(0_{X^{*}}, 0\right) \in \operatorname{cl} D \Rightarrow\left(0_{X^{*}}, 0\right) \in D, \\
\left(0_{X^{*}}, 0\right) \in \operatorname{cl} F \quad \Rightarrow \quad\left(0_{X^{*}}, 0\right) \in F,
\end{gathered}
$$

and the following statements:

(i) $x \in C, g(x) \in-K \Rightarrow f(x) \geq 0$,

(v) there exist $y^{*} \in K^{+}, x_{1}^{*} \in X^{*}$ and $x_{2}^{*} \in X^{*}$ such that

$$
f^{*}\left(x_{1}^{*}\right)+i_{C}^{*}\left(x_{2}^{*}\right)+\left(y^{*} \mathrm{og}\right)^{*}\left(-x_{1}^{*}-x_{2}^{*}\right) \leq 0,
$$

(vi) there exists $y^{*} \in K^{+}$such that

$$
f(x)+\left(y^{*} \circ g\right)(x) \geq 0, \forall x \in C .
$$

Then one has:
(a) (2.3) is equivalent to $[(\mathrm{i}) \Leftrightarrow(\mathrm{v})]$,
(b) (2.4) is equivalent to [(i) $\Leftrightarrow$ (vi)].

Proof. As in the proof of Theorem 2.1, one has (i) is equivalent to $\left(0_{X^{*}}, 0\right) \in \operatorname{cl} D$.

Moreover, it is easy to check that (v) is equivalent to $\left(0_{x^{*}}, 0\right) \in D$. Thus we get (a).

As shown in the proof of Theorem 2.1, (i) is equivalent to $\left(0_{X^{*}}, 0\right) \in \operatorname{epi}\left(f+i_{A}\right)^{*}$.

As epi $\left(f+i_{A}\right)^{*}=\mathrm{cl} F$ (see [3, Theorem 8.2]) we have (i) is equivalent to $\left(0_{x^{*}}, 0\right) \in \mathrm{cl} F$. Moreover, it is clear that (vi) is equivalent to $\left(0_{x^{*}}, 0\right) \in F$.

Therefore, one also gets (b). The proof is complete.

Corollary 2.1 [Farkas lemma for cone-convex systems] Consider the following conditions:

$$
\begin{gathered}
\left(0_{X^{*}}, 0\right) \in \mathrm{cl} D \Rightarrow\left(0_{X^{*}}, 0\right) \in D, \\
\left(0_{X^{*}}, 0\right) \in \mathrm{cl} F \quad \Rightarrow \quad\left(0_{X^{*}}, 0\right) \in F,
\end{gathered}
$$

and the following statements:

(i) $x \in C, g(x) \in-K \Rightarrow f(x) \geq 0$

(v) there exist $y^{*} \in K^{+}, x_{1}^{*} \in X^{*}$ and $x_{2}^{*} \in X^{*}$ such that

$$
f^{*}\left(x_{1}^{*}\right)+i_{C}^{*}\left(x_{2}^{*}\right)+\left(y^{*} \mathrm{o} g\right)^{*}\left(-x_{1}^{*}-x_{2}^{*}\right) \leq 0
$$


(vi) there exists $y^{*} \in K^{+}$such that

$$
f(x)+\left(y^{*} \circ g\right)(x) \geq 0, \forall x \in C .
$$

Then one has:
(a) (2.3) is equivalent to $[(\mathrm{i}) \Leftrightarrow(\mathrm{v})]$,

(b) (2.4) is equivalent to [(i) $\Leftrightarrow$ (vi)]

Proof. As in the proof of Theorem 2.1, one has (i) is equivalent to $\left(0_{X^{*}}, 0\right) \in \operatorname{cl} D$.

Moreover, it is easy to check that (v) is equivalent to $\left(0_{X^{*}}, 0\right) \in D$. Thus we get $(\mathrm{a})$.

As shown in the proof of Theorem 2.1, (i) is equivalent to $\left(0_{X^{*}}, 0\right) \in \operatorname{epi}\left(f+i_{A}\right)^{*}$.

As epi $\left(f+i_{A}\right)^{*}=\mathrm{cl} F$ (see [3, Theorem 8.2]) we have (i) is equivalent to $\left(0_{X^{*}}, 0\right) \in \mathrm{cl} F$. Moreover, it is clear that (vi) is equivalent to $\left(0_{x^{*}}, 0\right) \in F$.

Therefore, one also gets (b). The proof is complete.

Corollary 2.2 [Stable Farkas lemma for coneconvex systems] Consider the following conditions: epi $f^{*}+\bigcup_{y^{*} \in K^{+}} \operatorname{epi}\left(y^{*} \text { o } g\right)^{*}+$ epi $i_{C}^{*}$ is weak ${ }^{*}$ - closed

in $\quad X^{*} \in \mathbb{R}(2.5)$

U epi $\left(f+y^{*} \text { o } g+i_{C}\right)^{*}$ is weak ${ }^{*}$-closed in $X^{*} \times_{i}$.

Then we have

(c) (2.5) holds if and only if for any $x^{*} \in X^{*}$ and any $\beta \in \mathbb{R}$,

$$
\begin{gathered}
\left(x \in C, g(x) \in-K \Rightarrow f(x)-\left\langle x^{*}, x\right\rangle \geq \beta\right) \\
\mathrm{c} \\
\left(\exists y^{*} \in K^{+}, x_{1}^{*} \in X^{*} \text { and } x_{2}^{*} \in X^{*}\right. \text { such that } \\
\left.f^{*}\left(x_{1}^{*}\right)+i_{C}^{*}\left(x_{2}^{*}\right)+\left(y^{*} \circ g\right)^{*}\left(x^{*}-x_{1}^{*}-x_{2}^{*}\right) \leq-\beta\right) .
\end{gathered}
$$

(d) (2.6) holds if and only if for any $x^{*} \in X^{*}$ and any $\beta \in \mathbb{R}$,

$$
\left(x \in C, g(x) \in-K \Rightarrow f(x)-\left\langle x^{*}, x\right\rangle \geq \beta\right)
$$

$$
\exists y^{*} \in K^{+}: f(x)-\left\langle x^{*}, x\right\rangle+\left(y^{*} \mathrm{o} g\right)(x) \geq \beta, \forall x \in C .
$$

Proof. The proof is similar to that of Theorem 2.1 .

Remark 2.2 It is worth noting that (d) was given in [6]. Moreover, if we replace (2.5) by

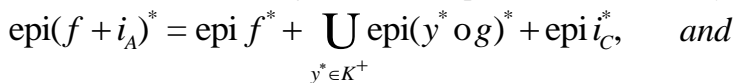

(2.6) by epi $\left(f+i_{A}\right)^{*}=\bigcup_{y^{*} \in K^{+}} \operatorname{epi}\left(f+y^{*} \mathrm{o} g+i_{C}\right)^{*}$ where $A:=C \cap g^{-1}(-K)$, then the conclusion of Corollary 2.2 still holds, and the assumptions on the closedness and the convexity of $C, f$, and $g$ can be removed.

\section{Asymptotic Farkas lemma for sublinear-convex systems}

Let $X, Y$ be lcHtvs, $C$ be a nonempty closed convex subset of $X, S: Y \rightarrow \mathbb{R} \cup\{+\infty\}$ be an lsc sublinear function and $g: X \rightarrow Y^{\bullet}$ be an $S$-convex mapping such that the set

$$
\{(x, y, \lambda) \in X \times Y \times \mathbb{R}: S(g(x)-y) \leq \lambda\}
$$

is closed in the product space $\mathrm{X} \times \mathrm{Y} \times \mathbb{R}$. Let us consider $f: X \rightarrow \mathbb{R} \cup\{+\infty\}$ and $\psi: \mathbb{R} \rightarrow \mathbb{R} \cup\{+\infty\}$ be proper convex lsc functions.

We now establish an asymptotic Farkas lemma for systems that are convex w.r.t. the sublinear function $S: Y \rightarrow \mathbb{R} \cup\{+\infty\}$.

Theorem 3.1 [Asymptotic Farkas lemma 2] Assume that the following condition holds: $(\operatorname{dom} f) \cap\{x \in C: \exists \alpha \in \operatorname{dom} \psi$ s.t. $(S \circ g)(x) \leq \alpha\} \neq \varnothing$.

Then the following statements are equivalent:

(a) $x \in C, \alpha \in \mathrm{i},(S \circ g)(x) \leq \alpha \Rightarrow f(x)+\psi(\alpha) \geq 0$

(b) there exist nets $\left(y_{i}^{*}, \gamma_{i}\right)_{i \in I} \subset Y^{*} \times \mathbb{R}_{+}$and $\left(x_{l i}^{*}, x_{2 i}^{*}, x_{3 i}^{*}, \varepsilon_{i}\right)_{i \in I} \subset X^{*} \times X^{*} \times X^{*} \times \mathbb{R} \times \mathbb{R}$ such with $y_{i}^{*} \leq \gamma_{i} S$ on $Y$ for all $i \in I$ such that $\varepsilon_{i} \geq f^{*}\left(x_{1 i}^{*}\right)+\left(y_{i}^{*} \mathrm{og}\right)^{*}\left(x_{2 i}^{*}\right)+i_{C}^{*}\left(x_{3 i}^{*}\right)+\psi^{*}\left(\eta_{i}+\gamma_{i}\right), \quad \forall i \in I$ and

$$
\left(x_{1 i}^{*}+x_{2 i}^{*}+x_{3 i}^{*}, \eta_{i}, \varepsilon_{i}\right) \rightarrow\left(0_{X^{*}}, 0,0\right)
$$

(c) there exist nets $\left(y_{i}^{*}, \gamma_{i}\right)_{i \in I} \subset Y^{*} \times \mathbb{R}_{+}$and $\left(x_{l i}^{*}, \eta_{i}, \varepsilon_{i}\right)_{i \in I} \subset X^{*} \times \mathbb{R} \times \mathbb{R}$ with $y_{i}^{*} \leq \gamma_{i} S$ on $Y$ for all $i \in I$ such that

$$
\begin{aligned}
& \varepsilon_{i} \geq\left(f+y_{i}^{*} \text { og }+i_{C}\right)^{*}\left(x_{i}^{*}\right)+\psi^{*}\left(\eta_{i}+\gamma_{i}\right), \quad \forall i \in I \\
& \text { and }\left(x_{i}^{*}, \eta_{i}, \varepsilon_{i}\right) \rightarrow\left(0_{X^{*}}, 0,0\right) .
\end{aligned}
$$

Proof. Let us set $\tilde{Y}=Y \times \mathbb{R}, \widetilde{Y}^{\bullet}=Y^{\bullet} \times$ $\mathbb{R}, \tilde{C}=C \times \mathbb{R}$ and set $\tilde{S}: \tilde{Y} \rightarrow \mathbb{R} \cup\{+\infty\}$ defined by $S(y, \lambda)=S(y)-\lambda$ for all $(\gamma, \alpha) \in \tilde{Y}$ Then $\tilde{C}$ is nonempty closed convex subset of $X \times \mathbb{R}, \tilde{S}$ is an lsc sublinear function. Let also $\widetilde{X}:=X \times \mathbb{R}$ 
$\tilde{g}=\tilde{X} \times \widetilde{Y}^{\bullet}$ and $\tilde{f}: \tilde{X} \rightarrow \mathbb{R} \cup\{+\infty\}$ be mappings defined by

$$
\begin{gathered}
\tilde{g}(x, \alpha):=(g(x), \alpha), \forall \in \tilde{X} \\
\text { and } \tilde{f}(x, \alpha)=f(x)+\psi(x), \forall(x, \alpha) \in \tilde{X}
\end{gathered}
$$

[(a) $\Rightarrow$ (b)] Assume that (a) holds. Since $f, \psi$ are proper lsc, convex functions, so is $\hat{f}$. Moreover, $\stackrel{\circ}{g}$ is $S$-convex as $g$ is $S$-convex. Now let $R$ be the closed convex cone defined by

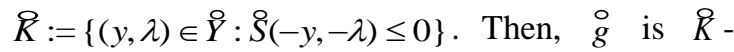
convex as well. The assumption (3.1) ensures that $\stackrel{\circ}{g}$ is $R$-epi closed while (3.2) guarantees $(\operatorname{dom} \hat{f}) \cap \mathcal{C} \cap \stackrel{\circ}{ }^{-1}(-R) \neq \varnothing$.

We now try to apply Theorem 2.1 with $\mathcal{X}, \vec{Y}, \mathcal{C}$, $\stackrel{\circ}{g}, \stackrel{f}{ }$, and $\mathcal{K}$ playing the roles of $X, Y, C, g$, $f$, and $K$, respectively.

From (a) and the definition of $f, \stackrel{\circ}{g}, \mathcal{C}, \mathcal{K}$, we have

$(x, \alpha) \in \mathcal{C}, \quad \stackrel{\circ}{g}(x, \alpha)=(g(x), \alpha) \in-\mathcal{K} \quad \Rightarrow \quad f(x, \alpha) \geq 0$,

which shows that (i) in Theorem 2.1 holds, and hence, there exist nets $\left(\mathscr{y}_{i}\right)_{i \in I} \subset \vec{K}^{+}$and $\left.\widetilde{\left(x_{l l}^{*}\right.}, \widetilde{x_{2 l}^{*}}, \widetilde{x_{3 l}^{*}}, \varepsilon_{i}\right)_{i \in I} \subset \widetilde{X^{*}} \times \widetilde{X^{*}} \times \widetilde{X}^{*} \times \mathbb{R}$ such that

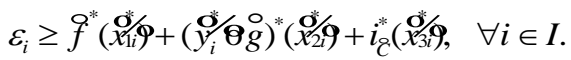

and

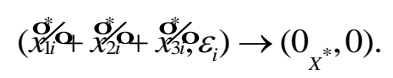

Since $\nabla^{*}=X^{*} \times_{i}$, there exist such $\left(x_{i i}^{*}, x_{2 i}^{*}, x_{i i}^{*}, \alpha_{i}, \beta_{i}, \delta_{i}\right)_{i \in I} \subset X^{*} \times X^{*} \times X^{*} \times \mathbf{i} \times \mathbf{i} \times \mathbf{i}$

such that $\left(\mathscr{X}_{i i}\right)_{i \in I}=\left(x_{i i}^{*}, \alpha_{i}\right)_{i \in I},\left(\mathscr{X}_{2 i} i_{i \in I}=\left(x_{2 i}^{*}, \beta_{i}\right)_{i \in I}\right.$ and $\left(x_{3 i t}^{*}\right)_{i \in I}=\left(x_{3 i}^{*}, \delta_{i}\right)_{i \in I}$. This and (3.6) imply that

$$
x_{1 i}^{*}+x_{2 i}^{*}+x_{3 i}^{*} \rightarrow 0_{X^{*}} \quad \text { and } \quad \alpha_{i}+\beta_{i}+\delta_{i} \rightarrow 0 .
$$

Moreover, since $\left(y_{i}\right)_{i \in I} \subset \hat{K}^{+}$, by Lemma 3.5 in [6], there exists a net $\left(y_{i}^{*}, \gamma_{i}\right)_{i \in I} \subset Y^{*} \times \mathbb{R}$ such that

$$
y_{i} \cong\left(y_{i}^{*},-\gamma_{i}\right), \gamma_{i} \geq 0
$$

and

$y_{i}^{*} \leq \gamma_{i} S$ on $Y$ for all $i \in I$.

By the definition of the conjugate function, for any $i \in I$, one has ([17, p.76])

$$
f^{*}\left(x_{i i}^{*}, \alpha_{i}\right)=f^{*}\left(x_{1 i}^{*}\right)+\psi^{*}\left(\alpha_{i}\right),
$$

$$
\begin{gathered}
\left(\mathscr{y}_{i}^{\circ} \otimes g\right)^{*}\left(x_{2 i}^{*}, \beta_{i}\right)=\sup _{x \in X, \alpha \in \mathrm{i}}\left\{\left\langle x_{2 i}^{*}, x\right\rangle+\beta_{i} \alpha-\left(y_{i}^{*} \mathrm{og}\right)(x)+\gamma_{i} \alpha\right\} \\
=\sup _{x \in X}\left\{\left\langle x_{2 i}^{*}, x\right\rangle-\left(y_{i}^{*} \mathrm{og}\right)(x)\right\}+\sup _{\alpha \in i}\left\{\left(\beta_{i}+\gamma_{i}\right) \alpha\right\} \\
= \begin{cases}\left(y_{i}^{*} \mathrm{o} g\right)\left(x_{2 i}^{*}\right) & \text { if } \beta_{i}=-\gamma_{i}, \\
+\infty & \text { otherwise, }\end{cases}
\end{gathered}
$$

and

$$
\begin{array}{r}
\underset{C}{i *}\left(x_{3 i}^{*}, \delta_{i}\right)=\sup _{x \in X, \alpha \in i}\left\{\left\langle x_{3 i}^{*}, x\right\rangle+\delta_{i} \alpha-i_{C}(x)\right\} \\
=\sup _{x \in X}\left\{\left\langle x_{3 i}^{*}, x\right\rangle-i_{C}(x)\right\}+\sup _{\alpha \in i}\left\{\delta_{i} \alpha\right\} \\
= \begin{cases}i_{C}^{*}\left(x_{3 i}^{*}\right) & \text { if } \delta_{i}=0, \\
+\infty & \text { otherwise. }\end{cases}
\end{array}
$$

(3.10)

Combining (3.5), (3.8), (3.9), and (3.10) we get (note that $\varepsilon_{i} \in \mathrm{i}$ for all $i \in I$ ):

$$
\varepsilon_{i} \geq f^{*}\left(x_{i j}^{*}\right)+\left(y_{i}^{*} \mathrm{og}\right)^{*}\left(x_{2 i}^{*}\right)+i_{C}^{*}\left(x_{3 i}^{*}\right)+\psi^{*}\left(\alpha_{i}\right)
$$

and $\beta_{i}=-\gamma_{i}, \delta_{i}=0$ for all $i \in I$, which together with (3.7) gives

$$
\alpha_{i}+\beta_{i}+\delta_{i}=\alpha_{i}-\gamma_{i} \rightarrow 0 .
$$

Set $\eta_{i}:=\alpha_{i}-\gamma_{i}$ for all $i \in I$. Then $\eta_{i} \rightarrow 0$ and the last inequality becomes

$\varepsilon_{i} \geq f^{*}\left(x_{1 i}^{*}\right)+\left(y_{i}^{*} \circ g\right)^{*}\left(x_{2 i}^{*}\right)+i_{C}^{*}\left(x_{3 i}^{*}\right)+\psi^{*}\left(\eta_{i}+\gamma_{i}\right), \quad \forall i \in I$.

Thus (b) is satisfied.

$[(b) \Rightarrow$ (c)] The same as the proof of $[(\mathrm{ii}) \Rightarrow$ (iii)] in Theorem 2.1 .

$[(\mathrm{c}) \Rightarrow$ (a)] Assume that (c) holds, i.e., there exist nets

$$
\left(y_{i}^{*}, \gamma_{i}\right)_{i \in I} \subset Y^{*} \times \mathbb{R}
$$

and $\left(x_{i}^{*}, \eta_{i}, \varepsilon_{i}\right)_{i \in I} \subset X^{*} \times \mathbb{R} \times \mathbb{R}$ with $y_{i}^{*} \leq \gamma_{i} S$ on $Y$ for all $i \in I$ such that (3.3) and (3.4) hold. It follows from (3.3) that $\varepsilon_{i} \geq\left\langle x_{i}^{*}, x\right\rangle-\left(f+y_{i}^{*} \mathrm{o} g\right)(x)+\left(\eta_{i}+\gamma_{i}\right) \alpha-\psi(\alpha)$, $\forall(x, \alpha) \in C \times \mathbf{i}, \quad \forall i \in I$,

or equivalently,

$$
\begin{gathered}
f(x)+\psi(\alpha)+\left(y_{i}^{*} \circ g\right)(x) \geq\left\langle x_{i}^{*}, x\right\rangle-\varepsilon_{i}+\eta_{i} \alpha+\gamma_{i} \alpha, \\
\forall(x, \alpha) \in C \times_{i}, \quad \forall i \in I
\end{gathered}
$$

(which still holds even in case $x \notin \operatorname{dom} f$, $x \notin \operatorname{dom} g$ and $\alpha \notin \operatorname{dom} \psi)$. 
Since $y_{i}^{*} \leq \gamma_{i} S$ on $Y$ for all $i \in I$, if $x \in C$ and $\alpha \in i \quad$ such that $(S \circ g)(x) \leq \alpha$, then $\left(y_{i}^{*} \circ g\right)(x) \leq \gamma_{i}(S \circ g)(x) \leq \gamma_{i} \alpha$ for all $i \in I$ (note that $\gamma_{i} \geq 0$ for all $\left.i \in I\right)$. So, for any $x \in C$ and $\alpha \in \mathrm{i}$ with $(S \circ g)(x) \leq \alpha$, (3.11) gives

$f(x)+\psi(\alpha)+\gamma_{i} \alpha \geq\left\langle x_{i}^{*}, x\right\rangle-\varepsilon_{i}+\eta_{i} \alpha+\gamma_{i} \alpha, \quad \forall i \in I$, which means that if $x \in C$ and $\alpha \in i$ with $(S \circ g)(x) \leq \alpha$, one has

$f(x)+\psi(\alpha) \geq\left\langle x_{i}^{*}, x\right\rangle-\varepsilon_{i}+\eta_{i} \alpha, \quad \forall i \in I$.

Passing to the limit both sides of the last inequality and taking the fact that (3.4) into account, we get (a). The proof is complete.

Theorem 3.2 [Asymptotic Farkas lemma 3] Assume that (3.2) holds. Then the following statements are equivalent:

(a)

$x \in C, \alpha \in \mathrm{i},(S \circ g)(x) \leq \alpha \Rightarrow f(x)+\psi(\alpha) \geq 0$

(d) there exists a net $\left(y_{i}^{*}, \gamma_{i}, \eta_{i}\right)_{i \in I} \subset Y^{*} \times i_{+} \times \mathbf{i}$ with $y_{i}^{*} \leq \gamma_{i} S$ on $Y$ for all $i \in I$ such that $\eta_{i} \rightarrow 0$, $\left(\eta_{i}+\gamma_{i}\right)_{i \in I} \subset \operatorname{dom} \psi^{*}$ and

$f(x)+\liminf _{i \in I}\left(\left(y_{i}^{*} \circ g\right)(x)-\psi^{*}\left(\eta_{i}+\gamma_{i}\right)\right) \geq 0, \forall x \in C$.

(3.12)

Proof. $[(a) \Rightarrow(d)]$ Assume that (a) holds. It follows from Theorem 3.1 that (c) holds. i.e., there exist nets $\quad\left(y_{i}^{*}, \gamma_{i}\right)_{i \in I} \subset Y^{*} \times i_{+} \quad$ and $\left(x_{i}^{*}, \eta_{i}, \varepsilon_{i}\right)_{i \in I} \subset X^{*} \times_{i} \times_{i}$ with $y_{i}^{*} \leq \gamma_{i} S$ on $Y$ for all $i \in I$ and such that

$\varepsilon_{i} \geq\left(f+y_{i}^{*} \mathrm{og}+i_{C}\right)^{*}\left(x_{i}^{*}\right)+\psi^{*}\left(\eta_{i}+\gamma_{i}\right), \quad \forall i \in I$, (3.13)

$[(\mathrm{c}) \Rightarrow(\mathrm{a})]$ Assume that (c) holds, i.e., there exist $\quad \operatorname{nets}\left(y_{i}^{*}, \gamma_{i}\right)_{i \in I} \subset Y^{*} \times i_{+}$and $\left(x_{i}^{*}, \eta_{i}, \varepsilon_{i}\right)_{i \in I} \subset X^{*} \times_{i} \times_{i}$ with $y_{i}^{*} \leq \gamma_{i} S$ on $Y$ for all $i \in I$ such that (3.3) and (3.4) hold. It follows from (3.3) that and $\left(x_{i}^{*}, \eta_{i}, \varepsilon_{i}\right) \rightarrow\left(0_{X^{*}}, 0,0\right)$.

By the definition of the conjugate function, (3.13) gives

rise

to

$\varepsilon_{i} \geq\left\langle x_{i}^{*}, x\right\rangle-\left(f+y_{i}^{*} \mathrm{og}+i_{C}\right)(x)+\psi^{*}\left(\eta_{i}+\gamma_{i}\right), \forall x \in X, \forall i \in I$.

Moreover, $\quad\left(\eta_{i}+\gamma_{i}\right)_{i \in I} \subset$ dom $\psi^{*}$, i.e., $\quad \psi^{*}\left(\eta_{i}+\gamma_{i}\right)$ attains finite value for all $i \in I$. So (3.15) is equivalent to

$f(x)+\left(y_{i}^{*} \mathrm{o} g\right)(x)-\psi^{*}\left(\eta_{i}+\gamma_{i}\right) \geq\left\langle x_{i}^{*}, x\right\rangle-\varepsilon_{i}, \forall x \in C, \forall i \in I$.

Taking the liminf in both sides of the last inequality (note also that (3.14) holds), we get

$f(x)+\underset{i \in I}{\liminf }\left(\left(y_{i}^{*} \circ g\right)(x)-\psi^{*}\left(\eta_{i}+\gamma_{i}\right)\right) \geq 0, \quad \forall x \in C$.

This means that (d) holds.

$[(d) \Rightarrow(a)]$. Assume that (d) holds, i.e., there exists a net $\left(y_{i}^{*}, \gamma_{i}, \eta_{i}\right)_{i \in I} \subset Y^{*} \times i_{+} \times \mathbf{i}$ with $y_{i}^{*} \leq \gamma_{i} S$ on $Y$ for all $i \in I$ such that $\eta_{i} \rightarrow 0$, $\left(\eta_{i}+\gamma_{i}\right)_{i \in I} \subset \operatorname{dom} \psi^{*}$ and (3.12) holds. Then from the definition of the conjugate function and (3.12), one gets

$f(x)+\liminf _{i \in I}\left(\left(y_{i}^{*} \circ g\right)(x)-\left(\eta_{i}+\gamma_{i}\right) \alpha+\psi(\alpha)\right) \geq 0, \quad \forall x \in C, \quad \forall \alpha \in \mathrm{i}$, which implies

$f(x)+\psi(\alpha)+\liminf _{i \in I}\left(\left(y_{i}^{*} \circ g\right)(x)-\eta_{i} \alpha-\gamma_{i} \alpha\right) \geq 0$,

$\forall x \in C, \forall \alpha \in \operatorname{dom} \psi$. (3.16)

According to Lemma 1.1 (iv) and the fact that $\eta_{i} \rightarrow 0$, we have

$\liminf \left(\left(y_{i}^{*} \circ g\right)(x)-\eta_{i} \alpha-\gamma_{i} \alpha\right)$

$=\liminf \left(\left(y_{i}^{*} \mathrm{o} g\right)(x)-\gamma_{i} \alpha\right)+\liminf \left(-\eta_{i} \alpha\right)$

$=\liminf _{i \in I}\left(\left(y_{i}^{*} \mathrm{o} g\right)(x)-\gamma_{i} \alpha\right), \forall x \in C, \forall \alpha \in \mathrm{i}$.

Combining this and (3.16), one gets $f(x)+\psi(\alpha)+\liminf _{i \in I}\left(\left(y_{i}^{*} \circ g\right)(x)-\gamma_{i} \alpha\right) \geq 0, \forall x \in C$,

$\forall \alpha \in \operatorname{dom} \psi$.

(Note that the last inequality still holds even $\alpha \notin \operatorname{dom} \psi)$.

Hence,

$f(x)+\psi(\alpha)+\liminf _{i \in I}\left(\left(y_{i}^{*} \circ g\right)(x)-\gamma_{i} \alpha\right) \geq 0, \forall x \in C, \forall \alpha \in \mathrm{i}$.

On the other hand, as $y_{i}^{*} \leq \gamma_{i} S$ on $Y$ for all $i \in I$, it follows that if $x \in C$ and $\alpha \in i$ such that $(S \circ g)(x) \leq \alpha$, then $\left(y_{i}^{*} \circ g\right)(x) \leq \gamma_{i}(S \circ g)(x) \leq \gamma_{i} \alpha, \quad \forall i \in I$, and hence, $\left(y_{i}^{*} \mathrm{o} g\right)(x)-\gamma_{i} \alpha \leq 0, \quad \forall i \in I$. 
So, for any $x \in C$ and $\alpha \in \mathrm{i}$ with $(S \circ g)(x) \leq \alpha$, we obtain from (3.17)

$f(x)+\psi(\alpha) \geq f(x)+\psi(\alpha)+\liminf _{i \in I}\left(\left(y_{i}^{*} \circ g\right)(x)-\gamma_{i} \alpha\right) \geq 0$, which is (a) and the proof is complete.

Set

$$
\begin{aligned}
& M:=\left\{\left(x^{*}, 0, r\right):\left(x^{*}, r\right) \in \operatorname{epi} f^{*}\right\}+\left\{\left(0_{X^{*}}, \eta, r\right):(\eta, r) \in \operatorname{epi} \psi^{*}\right\} \\
& +\bigcup_{\substack{y^{*} \in Y^{*}, \gamma \geq 0 \\
y^{*} \leq \gamma S}}\left\{\left(x^{*},-\gamma, r\right):\left(x^{*}, r\right) \in \operatorname{epi}\left(y^{*} \mathrm{o} g\right)^{*}\right\} \\
& +\left\{\left(x^{*}, 0, r\right):\left(x^{*}, r\right) \in \operatorname{epi} i_{C}^{*}\right\} \\
& N:=\left\{\left(0_{x^{*}}, \eta, r\right):(\eta, r) \in \operatorname{epi} \psi^{*}\right\} \\
& +\underset{\substack{* \\
y^{*} \in Y^{*}, \gamma \geq 0 \\
y^{*} \leq \gamma S}}{\mathrm{U}}\left\{\left(x^{*},-\gamma, r\right):\left(x^{*}, r\right) \in \operatorname{epi}\left(f+y^{*} \mathrm{o} g+i_{C}\right)^{*}\right\} .
\end{aligned}
$$

Corollary 3.1 [Farkas lemma for sublinear-convex systems] Assume that (3.2) holds. Consider the following conditions:

$$
\begin{aligned}
& \left(0_{x^{*}}, 0,0\right) \in \operatorname{cl} M \Rightarrow\left(0_{x^{*}}, 0,0\right) \in M, \\
& \left(0_{x^{*}}, 0,0\right) \in \operatorname{cl} N \Rightarrow \quad\left(0_{x^{*}}, 0,0\right) \in N,
\end{aligned}
$$

(3.18) $\left(\exists\left(y^{*}, \gamma\right) \in Y^{*} \times i_{,}, x_{1}^{*} \in X^{*}\right.$ and $x_{2}^{*} \in X^{*}$ such that $y^{*} \leq \gamma S$ on $Y$

and the following statements:

(a) $x \in C, \alpha \in \mathbf{i},(S \circ g)(x) \leq \alpha \Rightarrow f(x)+\psi(\alpha) \geq 0$

(b) there exist $\left(y^{*}, \gamma\right) \in Y^{*} \times \mathbf{i}_{+} \quad$ and $\left(x_{1}^{*}, x_{2}^{*}\right) \in X^{*} \times X^{*}$ with $y^{*} \leq \gamma S$ on $Y$ such that $0 \geq f^{*}\left(x_{1}^{*}\right)+i_{C}^{*}\left(x_{2}^{*}\right)+\left(y^{*} \mathrm{og}\right)^{*}\left(-x_{1}^{*}-x_{2}^{*}\right)+\psi^{*}(\gamma)$,

(c) there exist $\left(y^{*}, \gamma\right) \in Y^{*} \times x_{+}$, with $y^{*} \leq \gamma S$ on $Y$ such that $f(x)+\left(y^{*} \circ g\right)(x) \geq \psi^{*}(\gamma), \quad \forall x \in C$.

Then one gets

(i) (3.18) is equivalent to [ $(a) \Leftrightarrow(b)]$,

(ii) (3.19) is equivalent to $[(a) \Leftrightarrow(c)]$.
Proof. Set $\mathcal{X}, \stackrel{P}{Y}, \mathcal{C}, \stackrel{\circ}{g}, \stackrel{f}{f}$, and $R$ as in the proof of Theorem 3.1. The conclusion follows from Corollary 2.1 with $\mathcal{X}, \vec{Y}, \mathcal{C}, \stackrel{\circ}{g}, \mathcal{f}$, and $\mathcal{K}$ playing the roles of $X, Y, C, g, f$ and $K$, respectively.

Similar to Corollary 2.2, we get the following result.

Corollary 3.2 [Stable Farkas lemma for sublinear-convex systems] Assume that (3.2) holds. Consider the following statements:

(d) $M$ is weak ${ }^{*}$-closed in $X^{*} \times_{i} \times_{i}$.

(e) $N$ is weak ${ }^{*}$-closed in $X^{*} \times i \times i$.

(f) For any $x^{o} \cong\left(x^{*}, \eta\right) \in X^{*} \times i$ and any $\beta \in i$,

$\left(x \in C, \alpha \in \mathrm{i},(S \circ g)(x) \leq \alpha \Rightarrow f(x)+\psi(\alpha)-\left\langle x^{*}, x\right\rangle-\eta \alpha \geq \beta\right)$

(g) For any $x^{\prime}=\left(x^{*}, \eta\right) \in X^{*} \times \mathbf{i}$ and any $\beta \in \mathbf{i}$, $\left(x \in C, \alpha \in_{\mathrm{i}},(S \circ g)(x) \leq \alpha \Rightarrow f(x)+\psi(\alpha)-\left\langle x^{*}, x\right\rangle-\eta \alpha \geq \beta\right)$

$\left(\exists\left(y^{*}, \gamma\right) \in Y^{*} \times i_{+}\right.$such that $y^{*} \leq \gamma S$ on $Y$ and $\left.f(x)-\left\langle x^{*}, x\right\rangle+\left(y^{*} \circ g\right)(x) \infty \geq \psi^{*}(\gamma+\eta)+\beta, \forall x \in C\right)$.

Then we have [(d) $\Leftrightarrow$ (f)] and [(e) $\Leftrightarrow(\mathrm{g})]$.

Remark 3.1 It is worth noting that $[(\mathrm{e}) \Leftrightarrow(\mathrm{g})]$ was given in [6].

\section{Các bổ đề Farkas dạng tiệm cận cho các hệ lồi}

\section{- Nguyễn Định}

Trường Đại học Quốc tế, ĐHQG-HCM

\section{- Trần Hồng Mo}

Trường Đại học Tiền Giang

\section{TÓM TẮT}

Trong bài báo này chúng tôi thiết lập các điều kiện tuoong đưong (gọi là các đặc trung) của bao hàm thưc

$\{x \in X: x \in C, g(x) \in-K\} \subset\{x \in X: f(x) \geq 0\}$, trong đó $C$ là tập con lồi, đóng của không gian lồi địa phuong (kgldp) $X, K$ là nón lồi đóng trong 
kgldp $Y$, và $s: X \rightarrow Y$ là ánh $x a K$ - lồi, còn tập lồi đảo bên phải bao hàm thức trên được xác định bởi một hàm lồi, nửa liên tục duới $f$. Các đặc trung này được thiết lập mà không có bất kỳ điều kiện chính quy nào và thuoòng được gọi là các kết quả dạng Farkas tiệm cận (hay dạng xấp xỉ). Phần thứ hai của bài báo dành cho thiết lập các biến thể khác của bổ đề Farkas dạng tiệm cận cho ánh xạ $g$ là lồi theo một hàm duoói tuyến tính mở rộng $S$ (thay vì lồi theo nón $K$ nhu trên). Chúng tôi cũng

Tù khóa: Bổ đề Farkas, Bố đề Farkas theo dãy, giới hạn trên, giới hạn dưới.

\section{REFERENCES}

[1]. C.D. Aliprantis, K.C. Border, Infinite dimensional analysis, Springer-Verlag, Berlin, Germany (2006).

[2]. H.H. Bauschke, P.L. Combettes, Convex Analysis and Monotone Operator Theory in Hilbert Spaces, Springer, New York (2010).

[3]. R.I. Bot, Conjugate Duality in Convex Optimization, Springer-Verlag, Berlin (2010).

[4]. R.I. Bot, S.M. Grad, G. Wanka, New regularity conditions for strong and total FenchelLagrange duality in infinite dimensional, Nonlinear Analysis 69, 1, 323-336 (2008).

[5]. N. Dinh, M.A. Goberna, M.A. López, M. Volle, Convex inequalities without constraint qualification nor closedness condition, and their applications in optimization, Set-Valued Anal,. 18, 2540-2559 (2010) .

[6]. N. Dinh, M.A. Goberna, M.A. López, T.H. Mo, From the Farkas lemma to the Hahn-Banach theorem, SIAM J. Optim, 24, 2, , 678-701 (2014).

[7]. N. Dinh, V. Jeyakumar, G.M. Lee, Sequential Lagrangian conditions for convex programs with applications to semidefinite programming, J. Optim. Theory Appl. 125 (2005), 85-112.

[8]. N. Dinh, V. Jeyakumar, Farkas' lemma: Three decades of generalizations for mathematical optimization, Top, 22, 1-22 (2014).

[9]. N. Dinh, M.A. López, M. Volle, Functional inequalities in the absence of convexity and lower semicontinuity with applications to chứng minh rằng, duoói một số điè̀u kiện chính quy thích hợp, các kết quả đạt được ở trên cho lại các kết quả dạng Farkas hoặc dạng ổn định Farkas (stable Farkas lemmas) được thiết lập bởi nhiều tác giả trong nhũng năm gần đây, hoặc cho các phiên bản mói của các định lý này. Các kết quả đạt được có thể được sư dụng để nghiên cúu các bài toán tối uu mà ở đó các điều kiện chính quy không thỏa mãn. optimization, SIAM J. Optim. 20, 5, 423-445 (2010).

[10]. N. Dinh, T.H. Mo, Farkas lemma for convex systems revisited and applications to sublinearconvex optimization problems. Vietnam Journal of Mathematics, 43, 2, 297-321 (2015).

[11]. N. Dinh, B. Mordukhovich, T.T.A. Nghia, Subdifferentials of value functions and optimality conditions for DC and bilevel infinite and semi-infinite programs, Math. Program, 123, 101-138 (2010).

[12]. D.H. Fang, C. Li, K.F. Ng, Constraint qualifications for extebded Farkas's lemmas and Lagrangian dualities in convex infinite programming, SIAM J. Optim. 20, 1311-1332 (2010).

[13]. V. Jeyakumar, G.M. Lee, N. Dinh, New sequential Lagrange multiplier conditions characterizing optimality without constraint qualification for convex programming, SIAM J. Optim., 14, 2, 534-547 (2003).

[14]. R.E. Megginson, An Introduction to Banach Space Theory, Springer, New York (1998).

[15]. S. Simons, From Hahn-Banach to monotonicity, Springer-Verlag, Berlin (2007).

[16]. S. Simons, The Hahn-Banach-Lagrange theorem, Optimization, 56, 149-169 (2007).

[17]. Zà linescu, C., Convex Analysis in General Vector Spaces. World Scientific, River Edge, NJ (2002). 